\title{
Rechtsgeschichte
}

http://www.rg-rechtsgeschichte.de/rg7

Zitiervorschlag: Rechtsgeschichte Rg 7 (2005)

$\operatorname{Rg} 2005$

$169-171$

http://dx.doi.org/10.12946/rg07/169-171

\section{Gerrit Walther}

\section{Angry Old Man}


Überwältigten komme man nach Kramer zu einem »historischen Nullpunkt«. Die Position Amérys sei die »... diametral entgegensetzte(n) Position « zur Position Gellerts (449). Während Gellert die Autonomie des Subjekts unter der Folter behauptete, behaupte Améry die Überwältigung des Subjekts unter der Folter. Hier beziehe er seine Position explizit in Gegenüberstellung zu der Position Sartres, der auf den Widerstand der algerischen Widerstandskämpfer auch unter der Tortur abstellt: »Wer der Folter erlag, kann nicht mehr heimisch werden in der Welt « $(45 \mathrm{I})$. Mit der Folter finde die »irreversible Überwältigung eines Individuums « statt (473).

Mit Plausibilität kritisiert Kramer die These Amérys, indem er mit dessen Worten auf die Schwierigkeiten hinweist, die »Widerstandskraft « zu messen: "Noch keiner hat übersichtliche Grenzen ziehen können zwischen den sog. smoralischen` und der gleichfalls unter Anführungszeichen zu setzenden `körperlichen Widerstandskraft gegen physischen Schmerz« (452).

\section{Angry Old Man*}

Als vor mehr als zwanzig Jahren der erste Band von Harold J. Bermans Law and Revolution erschien, kam dies selbst einer Revolution gleich. Nicht mit der Renaissance nämlich, nicht mit dem Absolutismus und nicht mit I789 setzte der Rechtshistoriker aus Harvard The Formation of the Western Legal Tradition (I983; dt. I995), also die Entstehung des modernen Staates, an, sondern mit dem Sieg der Papstkirche im Investiturstreit zwischen I075 und I I22. Die entscheidenden Ergebnisse dieser Papal Revolution seien
Obwohl manchmal die nicht ausreichende Operationalisierung der durch Kramer methodisch entworfenen Diskursanalyse irritiert, so handelt es sich dennoch um ein sehr aufschlussreiches und "mit Herz und Kopf « geschriebenes Buch.

Resümierend kann man festhalten, dass Folter keine deutsche Rechtsgeschichte ist. Sie ist auch keine Geschichte bzw. keine naturgegebene Konstante der Gesellschaft. Folter dient grundsätzlich der Feststellung bzw. der Konstruktion der Wahrheit durch den Staat im Strafprozess. Insofern hat die Folter immer eine politischstaatliche Dimension. Ihre Verabsolutierung, Naturalisierung und Spektakularisierung lenkt den Blick von den Wahrheits- bzw. Machtstrategien $a b$, die der Staatsapparat durch sie verfolgt und durchsetzt. Es muss keinen Skandal darstellen, dass Literaten besser als Juristen diese Machtstrategien durchschauen, beschreiben und kritisieren.

\section{Domenico Siciliano}

das kanonische Recht gewesen, das elaborierteste, umfassendste seiner Zeit, und eine professionelle Methode seiner Handhabung, die Scholastik. Mit ihrer Hilfe habe die römische Kirche die Bevormundung durch weltliche Mächte abgestreift, ihren Supremat über die Laien fest etabliert, eine hoch effiziente Hierarchie geistlicher Juristen und Gerichte aufgebaut und die bis heute gültigen Prinzipien westlichen Rechts geprägt: die Idee, dass es immer weiter entwickelt werden müsse, dass seine Autorität über allen

\footnotetext{
* Harold J. Berman, Law and Revolution, II. The Impact of the Protestant Reformations on the Western Legal Tradition, Cambridge, Mass. and London: The Belknap Press of Harvard University Press 2003, XII, 522 S., ISBN 0-674-OII95-3
} 
anderen stehe und dass ihm daher auch der Mächtigste unterworfen bleibe.

In seinem neuen Werk beschreibt Berman, inzwischen Emeritus, die beiden nächsten großen Wendepunkte des Western legal system: die deutsche Reformation Martin Luthers und die Englische Revolution von I 640 bis I 689. Auf jeweils I70 Seiten folgen auf eine ereignisgeschichtliche Einführung jeweils Einzelkapitel über Legal Philosophy, Legal Science, Criminal Law, Civil and Economic Law und Social Law. Ein jedes ist eine souveräne Synthese der internationalen Forschungsliteratur - auch und gerade der deutschsprachigen: Germanica leguntur -, die in langen, aber oft geradezu spannenden Anmerkungen diskutiert und anhand einer klaren These ausgewertet wird: dass nämlich auch die neuere Geschichte des westlichen Rechts weit stärker vom jeweils herrschenden belief system geprägt worden sei, als heutige, säkular denkende Forscher wahrhaben wollen.

Von epochaler Bedeutung für die Rechtsgeschichte sei die Reformation vor allem der juristischen Konsequenzen wegen, die aus Luthers Aufwertung des Gewissens zur einzig möglichen Verbindung des Gläubigen mit Gott erwuchsen. Durch sie nämlich sei das Recht, dem diese Bedeutung bis dahin zugekommen sei, ganz auf die irdische Sphäre beschränkt und damit stark funktionalisiert worden. Dies habe zugleich bedeutet, dass alle irdischen Verhältnisse der Gläubigen untereinander nun rechtlicher Regelungen bedurften. Gesetze aber hätten nur noch den pragmatischen Zweck gehabt, die sündhaften Menschen mit allem Nachdruck an Vergehen zu hindern (die Herrschenden vor allem an Machtmissbrauch) und zur Erfüllung ihrer Pflichten gegenüber Gott und den Menschen anzuhalten. So wird Luther bei Berman zum eigentlichen Begründer jenes Positivismus, der Recht als Willensäußerung des Staates definiert. Das sehe wie Säkularisation aus, sagt er. Man könne es aber auch eine Spiritualisierung des weltlichen Rechts nennen $(64,349,369)$.

Ihre wichtigste Aufgabe nämlich hätten protestantische Theoretiker seit Melanchthon gerade darin gesehen, alles Recht auf dessen Übereinstimmung mit den biblischen Geboten hin zu prüfen. Kraft einer neuen Art von Topik, den loci communes, unterwarfen sie so die gesamte Jurisprudenz einer Systematisierung im Zeichen des Glaubens. Seien die Humanisten entweder in negativer Kritik an den Autoritäten der Scholastik verharrt (wie Valla) oder mit Rekonstruktionsversuchen des römischen Rechts befasst geblieben (wie Zasius und Alciat), so habe der usus modernus protestantorum (IO8) wie überhaupt die »Rezeption « darauf gezielt, alle konkurrierenden Rechte - das römische, die fürstlichen, feudalen, städtischen und kaufmännischen - auf einheitliche, gemeinsame Grundsätze zurückzuführen. Dahin habe einerseits die akademische Behandlung des Rechts durch einen professionellen Juristenstand gewirkt, andererseits die Gesetzgebung der Fürsten, deren Interessen eine solche Vereinheitlichung optimal entgegen kam. Sie behielten viele Regelungen des kanonischen Rechts bei, übernahmen es aber in ihre eigene, weltliche Regie.

In England kam die revolutionäre Entmachtung der Papstkirche nicht Fürsten zugute, sondern jenen bürgerlichen Korporationen, die seit I640 die Macht ergriffen. Diese echt calvinistische »strong emphasis placed on the corporate character of both action and thought « (305) manifestierte sich in der Abschaffung der königlichen prerogative courts zugunsten der common law courts, in epochalen Neuerungen im bürgerlichen, Handels- und Besitzrecht, aber auch in einer neuen Rechtsauffassung, die nur noch das 
als Recht akzeptierte, was die Gemeinschaft der Experten als solches anerkannte. Die ebenfalls calvinistische Gewissheit, dass die Engländer das von Gott auserwählte Volk seien, beflügelte den Stolz auf die nationale Rechtskontinuität - die englischen Revolutionäre behaupteten, zu vollenden, was Wilhelm der Eroberer begonnen habe, begünstigte aber auch die relativistische Einsicht, dass vernünftige Leute über Wahrheit sehr unterschiedlicher Meinung sein können. Von Max Webers "protestantischer Ethik « hingegen will Berman nichts wissen. Alles, was er darüber sage, sei auch bei Katholiken nachzuweisen (I 62).

Methodisch bekennt sich Berman mit provokanter Offenheit zur Tradition der Historischen Rechtsschule: was er mit belief system meine, entspreche ziemlich genau dem, was Savigny einst Volksgeist genannt habe (38I). Auch er ist überzeugt, dass das westliche Recht über zehn Jahrhunderte kontinuierlich vorangeschritten sei und zwar jeweils in enger Verbindung zum belief system. Immer dann, wenn die Diskrepanz zwischen beiden Sphären zu groß geworden sei, hätten revolutions dafür gesorgt, die Harmonie zwischen Glaubens- und Rechtssystem wiederherzustellen. Auch bei Berman also gibt es eine List der Vernunft - und einen Gang des Weltgeistes: Auf die Papal Revolution in Italien folgte die deutsche Reformation, dann der Englische Bürgerkrieg. Eine künftige Fortsetzung soll den deistischen Rationalismus als eigentliche French Revolution darstellen, dann die American Revolution: Part >English<, Part >French und schließlich die Russische Revolution von I9I7, da selbst der Bolschewismus als christliche Häresie dem Western belief system angehöre. Bis I9I4, letztlich sogar bis I989, habe der Westen ein (wenn auch nur »underneath the surface feststellbares) Ius commune besessen (I7).

Dass das christliche Moment inzwischen fast ganz aus dem westlichen Recht verschwunden ist, kann Berman historisch erklären. Doch es schmerzt ihn, weil es bewirkt habe, dass »our network of legal values lacks the power and vitality that it once had «(X). Das zeige gerade auch die akademische Rechtshistorie, die in übertriebener Spezialisierung den Blick für große Zusammenhänge verloren habe, sinnlose Epochentrennungen zwischen Mittelalter, Renaissance und Neuzeit vornehme und ohne Glauben, ohne Überzeugungen, ohne Botschaft ans Werk gehe.

Wem der nüchtern-säkulare Ton etwa von Michael Stolleis' Geschichte des öffentlichen Rechts als Norm vorschwebt, wird sich von dem Eifer, mit dem Berman ausgerechnet die Kirche, den klassischen Feind aller Aufklärer, zum Motor der Modernisierung und zum Garanten westlicher Rationalität erklärt, irritiert, vielleicht sogar pikiert fühlen. Der Verfasser weiß und will das. Ostentativ tritt er einer Gegenwart, die das Sprechen über »westliche Werte « für degoutant hält, als angry old man gegenüber, dem es nichts ausmacht, den Zorn von Strukturalisten, Kontingenztheoretikern, Mikrohistorikern und Eine-Welt-Enthusiasten auf sich $\mathrm{zu}$ ziehen. So ist auch sein neues Buch ein provokanter Alleingang, eine jener Meistererzählungen, die man mögen muss, wenn man es als das Meisterwerk würdigen will, das es ist.

Gerrit Walther 